\title{
Socio personal profile of resource poor dairy farmers and constraints in dairying
}

\author{
S. MAHALAKSHMI, M.C.A. DEVI AND R. KIRAN
}

KEY WORDS : Socio personal profile, Resource poor dairy farmers

HOW TO CITE THIS PAPER : Mahalakshmi, S., Devi, M.C.A. and Kiran, R. (2016). Socio personal profile of resource poor dairy farmers and constraints in dairying. Res. J. Animal Hus. \& Dairy Sci., 7(2) : 91-95 : DOI: 10.15740/HAS/RJAHDS/7.2/91-95.

Address for correspondence :

S. Mahalakshmi, Southern Regional Station ICAR-National Dairy Research Institute, Adugodi, BENGALURU (KARNATAKA) INDIA

Email : mahalucky32@gmail.com

Associated Authors':

M.C.A. Devi and R. Kiran, Southern Regional Station ICAR-National Dairy Research Institute, Adugodi, BENGALURU (KARNATAKA) INDIA 